\title{
The Cardiac Conduction System: A Mini Review
}

\author{
Daniel Jaffe*, Jennifer Hewit and Todd Crowder \\ Department of Physical Education, United States Military Academy, USA \\ *Corresponding author: Daniel Jaffe, Department of Physical Education, United States Military Academy, West Point, NY, USA
}

Submission: 眥 September 28, 2018; Published: 眥 October 15, 2018

\section{Editorial}

Biological membranes serve a number of functions with regard to regulating homeostasis i.e. a constant internal environment. First and foremost, the membrane serves as a means of protection and cellular integrity [1-3]. The membrane itself, composed primarily of a lipid bilayer consisting of a hydrophobic fatty-acid tail and a hydrophilic phospholipid head, prevents the undesired entrance of substances from the extracellular space that could potentially damage the vital organelles within the cell itself. Structure and shape of the exterior of the cell is maintained by the membrane, which sets up the outer boundaries of the cell [2-4].

The cell membrane is selectively permeable to various substrates, allowing these various substances to enter the cell through either passive or active transport [4]. Substances like water and diatomic oxygen are able to diffuse down their concentration gradients across the cell membrane. Integral membrane proteins, including ion channels, facilitate the movement of extracellular substances, including ions, into the intracellular space, and vice-versa, in order to maintain proper cellular functionality [2-4]. In addition, the cell membrane can invaginate and take in substrates (ligands) through the process of endocytosis. Endocytosis can be either selective, or non-selective i.e. pinocytosis. Exocytosis is the opposite process, where substrates produced within the cell are transported in vesicles to the exterior membrane of the cell, where the vesicle then fuses with the membrane, and the substrates are released into the extracellular space [5].

As mentioned previously, one of the functions of biological membranes is to maintain gradients [4]. Specifically, gradients can be electrical or chemical in nature, and are established through variations in concentration. A perfect example of an electrochemical gradient essential for the life processes is the balance established with the myocardium, specifically known as the cardiac conduction system [6].

The cardiac conduction system is a grouping of specialized myocardial cells responsible for the electrical signaling responsible for signaling the heart to contract $[1,6]$. On a cellular level within the heart, moving waves of depolarization and repolarization are caused by the influx and outflow of sodium, potassium, and calcium ions across the cell membrane $[1,6,7]$. While at rest, the interior of the cell contains a greater concentration of potassium, mediated via enhanced permeability to potassium relative to sodium, and active removal of sodium to maintain a resting potential of $-90 \mathrm{mV}[1,7]$. The negativity of the interior of the cell allows the cardiac tissues to display the characteristic of excitability, or ability to respond to an electrical stimulus by producing a mechanical event $[1,7,8]$.

The rapid influx of sodium, initiated by calcium shifting, yields a dramatic increase in charge within the cell from $-90 \mathrm{mV}$ up to $+30 \mathrm{mV}$, propagating the movement of an action potential across the myocardium, from the epicardium down to the endocardium during phase 0 of the cardiac cycle $[7,8]$. Repolarization, or restoration of the resting potential, occurring from the endocardium to the epicardium, takes place when the potassium channels open and potassium rushes out of the cell, with sodium being actively removed to return the interior of the cell to $-90 \mathrm{mV}$, demonstrated by phases $1-4$ of the cardiac cycle $[7,8]$.

As the cardiac muscle functions as a syncytium, contracting simultaneously due to the rapid conduction of the electrical stimuli across gap junctions, each of these ionic movements can be identified on the ECG tracing $[8,9]$. Through observing duration, amplitude, and configuration of the ECG tracing, it becomes possible to observe the electrical activity of the heart [8].

The heart itself is comprised of four separate and distinct chambers: the left and right atria and ventricles [7,10]. Blood flows into the heart through the right atrium, originating from systemic circulation via the inferior and superior vena cava and coronary sinus. Travelling from the right atrium, the blood passes through the tricuspid atrioventricular (AV) valve into the right ventricle $[7,10]$. From the right ventricle, the deoxygenated blood is pumped through the pulmonary semilunar valve into the pulmonary trunk for transportation to the lungs for reoxygenation. The trunk divides into two pulmonary arteries, one carrying blood to each the right and left lung, where they continue to branch into capillaries for gas exchange with the alveoli of the lungs $[7,10]$.

Upon oxygenation, the blood that was pumped to the lungs returns to the heart via the pulmonary veins, where it is received by 
the left atrium. Once in the left atrium, the freshly oxygenated blood travels through the bicuspid, or mitral, atrioventricular valve into the left ventricle $[7,8,10]$. Blood in the left ventricle is then expelled through the aortic semilunar valve into the aorta, where it is then further distributed into systemic circulation, delivering oxygen and nutrients to the working and non-working tissues of the body. The quantity of blood that is supplied to various body parts depends upon the metabolic demands of the tissues, specifically the demands implicated during exercise relative to those observed during the resting state [7]

The annulus fibrosus, a fibrous connective tissue, is responsible for separating the atria and ventricles, as well as providing scaffolding for attachment of the papillary muscles and other myocardial tissue, maintaining the physical structure of the heart [10]. In addition to providing a skeletal frame for muscular insertions, the annulus fibrosus conducts electrical currents down the atrioventricular (AV) node, which is located near the interatrial septum, or the wall of myocardium separating the right atrium from the left $[8,10]$. This electrical impulse represents the mean electrical vector, originating at the AV node and travelling inferiorly and to the left in the frontal plane $[8,11]$. This vector is represented as an upward wave deflection if the charge is moving towards a positive electrode, typically seen in V5 \& V6; a downward deflection if it moves away from the electrode in V1 \& V2; and a biphasic wave if it moves perpendicular to the electrode, typically V3 \& V4 $[8,11,12]$. Dividing the two halves of the heart is the interventricular septum [10]. This septum functions as the primary physical barrier dividing the left from the right ventricle. This septum also functions as the housing unit for the bundle branches which are responsible for transmitting the electrical signals to the apex of the heart and up to the Purkinje fibers, maintaining synchronization in ventricular contractions $[8,10]$.

In addition to the basic anatomical differences observed between the left and right side of the heart attributed to destination of flow output, there exist variations in electrophysiological composition between the two halves of the heart $[8,10]$. The sinoatrial, or SA, node is comprised of a group of specialized myocardial cells located in the posterior wall of the right atrium. It is here that the impulse for normal heart contractions is initiated. The SA node, also known as the heart's pacemaker, establishes a sinus rhythm of approximately 100 beats per minute. It is through the introduction of autonomic nervous system activity, both sympathetic and parasympathetic, that heart rate can be altered $[10,13]$.

From here, the electrical impulse then travels across the atrial septum, reaching the atrioventricular, or AV, node, located posteroinferior region of the interatrial septum, signaling the atria to contract in near simultaneous fashion $[8,11]$. The $\mathrm{P}$ wave is representative of atrial depolarization [8]. The AV node transmits the electrical impulse from the atria into the ventricles, creating a delay of approximately $0.10 \mathrm{sec}$ before allowing the signal to enter the atrioventricular bundle, or bundle of His. This time lapse permits complete emptying of the atria into the ventricles. The "atrial kick," or active contraction of the atria, completes atrial emptying that was not accomplished via passive movement of blood from the atrium to the ventricle $[8,11]$.

The electrical impulse, in accordance with the mean electrical vector mentioned previously, travels down the ventricular septum to the apex of the heart. Upon reaching the apex, branches travel through and up both the right and left ventricular walls, permitting signal transmission. The terminal ends of these branches are known as the Purkinje fibers [14]. Electrical impulses travel through the ventricles approximately six times faster than through the rest of the cardiac conduction system to allow simultaneous contraction of the left and right ventricles given the presence of gap junctions. The QRS segment represents ventricular depolarization. The T-wave, or final wave in the cardiac cycle, is representative of repolarization, occurring from endocardium to epicardium [8]. Upon completion of repolarization, the process is prepared to begin again.

\section{References}

1. Klaeboe LG, Brekke PH, Lie OH, Aaberge L, Haugaa KH, et al. (2018) Classical mechanical dyssynchrony is rare in transcatheter aortic valve implantation-induced left bundle branch block. European Heart Journal-Cardiovascular Imaging Vol. 127.

2. Alberts B, Bray D, Hopkin K, Johnson A, Lewis J, et al. (2009) Essential cell biology ( $3^{\text {rd }}$ edn), Garland Science, Florence, Italy.

3. Halfter W, Oertle P, Monnier CA, Camenzind L, Reyes LM, et al. (2015) New concepts in basement membrane biology. The Federation of European Biochemical Societies Journal 282(23): 4466-4479.

4. Robertson JL (2018) The lipid bilayer membrane and its protein constituents. The Journal of General Physiology 150(10).

5. Wu LG, Hamid E, Shin W, Ciang HC (2014) Exocytosis and endocytosis: Modes, functions, and coupling mechanisms. Annual Review of Physiology 76: 301-331.

6. Curran J, Makara MA, Little SC, Musa H, Liu B, et al. (2014) EHD3-dependent endosome pathway regulates cardiac membrane excitability and physiology. Circulation Research 115(1): 68-78.

7. Astrand PO, Rodahl K, Dahl HA, Stromme SB (2003) Textbook of work physiology: Physiological bases of exercise ( $4^{\text {th }}$ edn). Human Kinetics Publishers Inc, Champaign, Illinois, USA.

8. Goldberger AL (2006) Clinical electrocardiography. Mosby, Philadelphia, Pennsylvania, USA.

9. Carette D, Gilleron J, Denizot JP, Grant K, Pointis G, et al. (2015) New cellular mechanisms of gap junction degradation and recycling. Biology of the Cell 107(7): 218-231.

10. Opfer E, Shah S (2018) Advances in pediatric cardiovascular imaging. Missouri Medicine 115(4): 354-360.

11. Billette J, Tadros R (2014) Integrated rate-dependent and dual pathway AV nodal functions: Principles and assessment framework. American Journal of Physiology: Heart and Circulation Physiology 306(2): 173183.

12. Magee C, Kazman J, Haigney M, Oriscello R, DeZee KJ, et al. (2014) Reliability and validity of clinician ECG interpretation for athletes. Annals of Noninvasive Electrocardiography 19(4): 319-329.

13. Kashou AH, Kashou HE (2017) Physiology, sinoatrial node (SA node). Stat Pearls.

14. Sedmera D, Gourdie RG (2014) Why do we have Purkinje fibers deep in our heart? Physiological Research 63(Suppl 1): S9-18. 
Creative Commons Attribution 4.0 International License

For possible submissions Click Here

Submit Article
BOJTS|
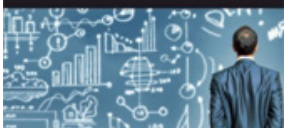

-

-

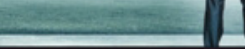

COJ Technical \& Scientific Research Benefits of Publishing with us

- High-level peer review and editorial services

- Freely accessible online immediately upon publication

- Authors retain the copyright to their work

- Licensing it under a Creative Commons license

- Visibility through different online platforms 